\title{
BOUNDS FOR THE ORDER OF SUPERSOLUBLE AUTOMORPHISM GROUPS OF RIEMANN SURFACES
}

\author{
REZA ZOMORRODIAN
}

(Communicated by B. Srinivasan)

\begin{abstract}
The maximal automorphism groups of compact Riemann surfaces for a class of groups positioned between nilpotent and soluble groups is investigated. It is proved that if $G$ is any finite supersoluble group acting as the automorphism group of some compact Riemann surface $\Omega$ of genus $g \geq 2$, then:

(i) If $g=2$ then $|G| \leq 24$ and equality occurs when $G$ is the supersoluble group $D_{4} \otimes Z_{3}$ that is the semidirect product of the dihedral group of order 8 and the cyclic group of order 3 . This exceptional case occurs when the Fuchsian group $\Gamma$ has the signature $(0 ; 2,4,6)$, and can cover only this finite supersoluble group of order 24 .

(ii) If $g \geq 3$ then $|G| \leq 18(g-1)$, and if $|G|=18(g-1)$ then $(g-1)$ must be a power of 3 . Conversely if $(g-1)=3^{n}, n \geq 2$, then there is at least one surface $\Omega$ of genus $g$ with an automorphism group of order $18(g-1)$ which must be supersoluble since its order is of the form $2 \cdot 3^{m}$. This bound corresponds to a specific Fuchsian group given by the signature $(0 ; 2,3,18)$. The terms in the chief series of each of these Fuchsian groups to the point where a torsion-free subgroup is reached are computed.
\end{abstract}

\section{INTRODUCTION}

The comprehensive work of C. H. Sah is a landmark paper [8]. In addition to considering soluble groups as one class and establishing a necessary and sufficient condition for a Fuchsian group to cover a finite soluble group, he showed that several of the sporadic finite simple groups, such as the small Janko group, are "Hurwitz groups". Sah made a breakthrough by defining the " $p$-periods" of a Fuchsian group $\Gamma$ for any prime $p$ which divides the products of the periods. He also stated that, since the "Hurwitz groups" (the maximal automorphism groups of order $84(g-1))$ always have simple non-abelian homomorphic image and cannot be soluble, the maximal order of the soluble

Received by the editors August 24, 1987 and, in revised form, June 30, 1988 and January 15, 1989.

1980 Mathematics Subject Classification (1985 Revision). Primary 20H10, 20D15, 20 D45.

Key words and phrases. Fuchsian groups, supersoluble automorphism groups, nilpotent automorphism groups, maximal automorphism groups, compact Riemann surfaces, action of groups, generators, relations, signatures, bounds, chief series. 
automorphism group must be $48(g-1)$. This upper bound occurs when $\Gamma$ is the group of the signature $(0 ; 2,3,8)$. In a later paper entitled "Residual Nilpotency of Fuchsian Groups", A. M. Macbeath [7] took this process a significant step further by defining for each Fuchsian group $\Gamma$ a $p$-localization group $\Gamma_{p}$, which was also, in general, a Fuchsian group. In a paper entitled "Nilpotent Automorphism Groups of Riemann Surfaces", this author [12] used the methods of Siegel and Macbeath to present a complete solution to the "nilpotent" analogue of Hurwitz' theorem and obtained the bound $16(g-1)$ for the order of the maximal nilpotent automorphism groups. The groups of maximal order turned out to be all 2-groups, i.e., those which had a power of 2 for an order. The present paper studies yet another class of automorphism groups of Riemann surfaces of $g \geq 2$ called supersoluble automorphism groups. These groups are positioned between nilpotent and soluble groups and are finite and soluble. Since the derived group of a finite supersoluble group is nilpotent [11] and a finite nilpotent group is the direct product of its Sylow-subgroups, the abelianizing homomorphism which factors out the derived group $\Gamma^{\prime}$ of $\Gamma(S)$ and which contains the maximal abelian factor group of $\Gamma$ plays a key role in this investigation of supersoluble automorphism groups. Moreover, a theorem of D. Singerman [10] often has been used in computing the signature of the derived group $\Gamma^{\prime}$ of $\Gamma$. The results are as follows: Suppose $G$ is any finite supersoluble group of automorphisms of some Riemann surface $\Omega$ of genus $g \geq 3$. Then $|G| \leq 18(g-1)$, and if $|G|=18(g-1)$ then $(g-1)$ must be a power of 3 . Conversely, if $(g-1)=3^{n}$ then there is at least one surface $\Omega$ of genus $g$ with an automorphism group of order $18(g-1)$ which must be supersoluble since it has an order of the form $2 \cdot 3^{m}$. This bound corresponds to a Fuchsian group given by the signature $(0 ; 2,3,18)$. It will be proven that the supersoluble groups take their upper bound among the $(2,3, n)$ triangle groups with the exception of one group of low order when $\Gamma$ has the signature $(0 ; 2,4,6)$ which can only cover one group of order 24 . It will also be proven that, among the $(2,3, n)$ triangle groups, only the $(2,3,6 r)$ groups should be considered. Oddly enough, the only candidates happen to be the two triangle groups $(2,3,12)$ and $(2,3,18)$, both with nilpotent-admissible derived groups of signatures $(1 ; 2)$ and $(1 ; 3)$, respectively, along with extremely similar structures in their derived series. Although these two groups both admit factor groups with Sylow tower properties, only $(2,3,18)$ is supersoluble.

\section{Notations}

Let $\Gamma$ as usual be any cocompact Fuchsian group, that is one with the quotient space $H^{2} / \Gamma$, where $H^{2}$ is the complex upper-half plane and $\Gamma$ has the presentation

$$
\Gamma=\left\langle a_{1}, b_{1}, \ldots, a_{g}, b_{g}, x_{1}, \ldots, x_{r} \mid x_{i}^{m_{i}}, \prod_{1}^{r} x_{i} \prod_{i}^{g}\left[a_{j}, b_{j}\right]\right\rangle
$$


for all $i=1,2, \ldots, r$, where $g \geq 0, r \geq 0, m_{i} \geq 2$, and $[a, b]=a b a^{-1} b^{-1}$, see $[2,7,12,13]$. We then say $\Gamma$ has signature

$$
S=\left(g ; m_{1}, \ldots, m_{r}\right) \text {. }
$$

The integers $m_{i}$ 's are called the periods and $g$ is called the genus. If $r=0$, $\Gamma$ will be called a surface group denoted by the signature $\left(g\right.$; ). If all $m_{i} \geq 2$, $S$ is said to be reduced, otherwise nonreduced. If $\Gamma$ has signature $S$, we write $\Gamma=\Gamma(S)$. If $g=0$ and $r=3$ we call $(0 ; l, m, n)$ a triangle group. The rational numbers:

$$
\begin{gathered}
\chi(S)=2-2 g+\sum_{i=1}^{r}\left(\frac{1}{m_{i}}-1\right) \\
Y=-\chi(S)
\end{gathered}
$$

are called the Euler characteristic, and its negative, of the signature $S$. The signature $S$ is called degenerate if its reduced form satisfies one of the following:

(a) $g=0$ and $r=1$ or

(b) $g=0$ and $r=2, m_{i} \neq m_{2}$,

otherwise $S$ will be called nondegenerate. For each $i=1, \ldots, r$, let $p^{\alpha_{i}}$ be the highest power of the prime $p$ which divides $m_{i}$. We then call the signature

$$
S_{p}=\left(g ; p^{\alpha_{1}}, \ldots, p^{\alpha_{r}}\right)
$$

the $p$-localization of $S$ [7]. If every period of $S$ is already some power of one fixed prime $p$ we then call $S$ a $p$-local signature. We call the signature $S$ nilpotent-admissible if every $p$-local signature $S_{p}$ of $S$ is nondegenerate. If $S$ is nondegenerate and $\Gamma_{1}$ is a subgroup of finite index in $\Gamma(S)$, then there exists a signature $S_{1}$ such that $\Gamma_{1}=\Gamma\left(S_{1}\right)$ and has index given by the formula

$$
\left[\Gamma: \Gamma_{1}\right]=\chi\left(S_{1}\right) / \chi(S) \text {. }
$$

A homomorphism $f$ from a Fuchsian group $\Gamma(S)$ onto a finite group $G$ which preserves all the periods of $\Gamma$, i.e., for every $x_{i}$ of order $m_{i}$ the order of $f\left(x_{i}\right)$ is precisely $m_{i}$, will be called smooth. If $p$ is a prime number then $f$ is called $p$-smooth if the order of $f\left(x_{i}\right)$ is divisible by the highest power $p^{\alpha_{i}}$ of $p$ which divides $m_{i}$. If $f$ is smooth then $\operatorname{ker}(f)$ will be a Fuchsian surface group. A finite group $G$ which has such a smooth homomorphism onto it will be called a smooth quotient group of $\Gamma$. A homomorphism of particular importance in this paper is the abelianizing homomorphism $\theta$ which factors out the derived group $\Gamma^{\prime}=[\Gamma: \Gamma]$ of $\Gamma(S)$, and its factor group is the maximal factor group of $\Gamma$ of abelian nature. Thus if $\Phi: \Gamma(S) \rightarrow G$ is any smooth homomorphism mapping $\Gamma(S)$ onto any finite group $G$, then $\Phi$ can always be regarded as the composition of $\theta$ with some other homomorphism $\Psi$ which maps $\Gamma / \Gamma^{\prime}$ onto $G$. Moreover the composition $\Psi \circ \theta$ will have a torsion free kernel. 


\section{SOME BASIC EXISTING RESULTS ON FINITE SUPERSOLUBLE GROUPS}

In this section we merely state some definitions and theorems on finite supersoluble groups. For a proof of any of the theorems the reader is referred to M. Weinstein [11]. Since in the next sections where the main results are obtained the reader is often referred to some existing results on finite supersoluble groups we devoted this section for reference simply to facilitate the work of the readers.

Definition 2.1. If $G$ is any finite group and $G_{1}$ and $G_{2}$ are normal subgroups of $G$ with $G_{1} \leq G_{2}$, then $G_{2} / G_{1}$ is called a chief factor or principal factor of $G$ if there is no normal subgroup $G_{3}$ of $G$ such that

$$
G_{1}<G_{3}<G_{2} .
$$

Definition 2.2. A finite group $G$ is said to be supersoluble whenever all the chief factors in any chief series of $G$ are cyclic of prime order.

Theorem 2.3. (i) Every subgroup of a supersoluble group is supersoluble.

(ii) Every homomorphic image of a supersoluble group is supersoluble.

(iii) The direct product of any two supersoluble groups is supersoluble.

Theorem 2.4. Let $G$ be any finite group with a normal cyclic subgroup $G_{1}$ such that $G / G_{1}$ is supersoluble. Then $G$ is supersoluble.

Definition 2.5. Let $G$ be a finite group and let $p_{1}<p_{2}<\cdots<p_{r}$ be the distinct primes dividing the order of $G$. Then $G$ is said to satisfy the Sylow tower property if, for the $p_{i}$-Sylow subgroups $G_{p_{1}}, G_{p_{2}}, \ldots, G_{p_{r}}$ of $G$, we have

$$
G_{p_{r}} G_{p_{r-1}} \ldots G_{p_{k}} \text { is normal in } G \text { for all } k=1,2,3, \ldots, r \text {. }
$$

Definition 2.6. Let $p$ be any prime. A group $G$ is said to be strictly $p$-closed whenever $G_{p}$, a Sylow $p$-subgroup of $G$, is normal and when $G / G_{p}$ is abelian of the exponent dividing $p-1$.

Theorem 2.7. The derived group $G^{\prime}=[G, G]$ of a finite supersoluble group $G$ is a nilpotent group.

Theorem 2.8. Every maximal subgroup of a finite supersoluble group $G$ has a prime index in $G$.

Theorem 2.9. Every group $G$ which is strictly p-closed is supersoluble.

Theorem 2.10. The group $G$ is supersoluble if and only if

(i) $G$ satisfies the Sylow tower property and

(ii) For every Sylow p-subgroup $G_{p}$ of $G$, the quotient group $N_{G}\left(G_{p}\right) /$ $C_{G}\left(G_{p}\right)$ is strictly p-closed, where $N_{G}$ and $C_{G}$ are the normalizer and centralizer respectively. 


\section{BOUND FOR THE ORDER OF SUPERSOLUBLE GROUPS OF AUTOMORPHISMS}

In this section we study another class of automorphism groups of a compact Riemann surface called supersoluble automorphism groups. We consider all those homomorphisms of Fuchsian groups targeting the finite supersoluble groups, and find an upper bound for these target groups. We also obtain some results on the supersoluble smooth quotient groups. But first the following two theorems give some relationships between finite supersoluble groups and cocompact Fuchsian groups.

Theorem 3.1. Let $\Gamma$ be a finitely generated cocompact Fuchsian group with signature $S=\left(\gamma ; m_{1}, m_{2}, \ldots, m_{r}\right)$. Let $G$ be a finite supersoluble group covered by $\Gamma(S)$. Then $\Gamma^{\prime}=[\Gamma, \Gamma]$ the derived group of $\Gamma$ has a nilpotent-admissible signature.

Proof. Let $\phi: \Gamma(S) \rightarrow G$ be a smooth homomorphism onto $G$. Abelianizing both $\Gamma$ and $G$, and letting $\delta: \Gamma \rightarrow \Gamma / \Gamma^{\prime}$ and $\theta: G \rightarrow G / G^{\prime}$ (where $\Gamma^{\prime}=[\Gamma, \Gamma]$ and $G^{\prime}=[G, G]$ are the commutator subgroups of $\Gamma$ and $G$ respectively) be the two canonical homomorphisms. Then there must exist another homomorphism $\psi: \Gamma / \Gamma^{\prime} \rightarrow G / G^{\prime}$ such that the following diagram commutes:

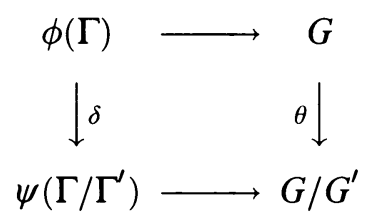

that is $\theta \circ \phi=\psi \circ \delta$. Clearly if $\gamma \in \Gamma(S)$ and $\phi(\gamma)=g \in G$, then $\delta(\gamma)=$ $\gamma \Gamma^{\prime}$ and $\theta(g)=g G^{\prime}$. Therefore if we define $\psi$ by $\psi\left(\gamma \Gamma^{\prime}\right)=\phi(\gamma) G^{\prime}$ where $\phi(\gamma) \in G$, then we have $\psi(\delta(\gamma))=g G^{\prime}=\theta(g)=\theta(\phi(\gamma))$, which is true for all $\gamma \in \Gamma(S)$. Now letting $\gamma=1$, that is, the identity of $\Gamma(S)$, we obtain $\psi\left(\Gamma^{\prime}\right)=G^{\prime}$. This proves that the homomorphism $\psi$ is a covering from $\Gamma^{\prime}$ onto the derived group $G^{\prime}$ of $G$. By theorem (2.7) of the last section, $G^{\prime}$ is nilpotent, that is, $\Gamma^{\prime}$, the derived group of $\Gamma$, can cover at least one nilpotent Riemann surface automorphism group. Hence by a theorem of Macbeath [7, p. 309] $\Gamma^{\prime}$ must have a nilpotent-admissible signature, i.e., one whose $p$-local signatures are all nondegenerate.

Theorem 3.2. Let $S=\left(0 ; m_{1} \ldots, m_{r}\right)$ be a signature of genus 0 and $G$ a finite supersoluble automorphism group covered by $\Gamma(S)$. Let $\Pi(S)$ denote the set of prime factors of $m_{1} m_{2} \ldots m_{r}$, and let $p_{1}=\min \{\Pi(S)\}$, we have:

(i) $p_{1}$ is the smallest prime divisor of order of $G$ and the Sylow $p_{1}$-subgroup of $G$ is a smooth factor group of $\Gamma(S)$.

(ii) The $p_{1}$-localization of the signature $S$ is nondegenerate.

Proof. Let $q_{1}<q_{2}<\cdots<q_{k}$ be the distinct prime factors of the order of $G$. Since $G$ is supersoluble it must satisfy the Sylow-tower property, i.e., for 
the Sylow $q_{i}$-subgroups $G_{q_{1}}, \ldots, G_{q_{k}}$, the group $N=G_{q_{k}} G_{q_{k-1}} \cdots G_{q_{2}}$ must be normal in $G . G / N$ is a factor group of $G$. Thus there exists a homomorphism $F: G \rightarrow G / N$ whose kernel has an order relatively prime to $q_{1}$. Next, since $\Gamma(S)$ covers $G$ there exists a smooth homomorphism $\Phi: \Gamma(S) \rightarrow G$ onto $G$. Therefore the composition of $F$ and $\Phi$ is a homomorphism $(F \circ \Phi): \Gamma(S) \rightarrow$ $G_{q_{1}}$ which is again smooth. This shows that $G_{q_{1}}$ is a homomorphic image of $\Gamma(S)$, and by Theorem 5.3 [7, p. 304] there exists a unique homomorphism $\Phi_{q_{1}}: \Gamma\left(S_{q_{1}}\right) \rightarrow G_{q_{1}}$ such that $F \circ \Phi=\Phi_{q_{1}} \circ L_{q_{1}}$ where $L_{q_{1}}: \Gamma(S) \rightarrow \Gamma\left(S_{q_{1}}\right)$ is the $q_{1}$-localization homomorphism [7]. This proves $G_{q_{1}}$ is a smooth homomorphic image of $\Gamma\left(S_{q_{1}}\right)$. Therefore, $q_{1} \in \Pi(S)$, because if $q_{1} \notin \Pi(S)$ the $q_{1}$-localization of $\Gamma(S)$ is trivial (see Theorem 2.11 [12, p. 252]). This only applies because $S$ has genus 0 , hence $q_{1} \geq p_{1}$. On the other hand, since $\Phi$ is a smooth homomorphism of $\Gamma(S)$ onto $G, G$ must contain elements of order $p_{1}$ and so $q_{1} \leq p_{1}$ which implies $q_{1}=p_{1}$ and proves (i). Next, since $q_{1}=p_{1}$ and $\Phi_{p_{1}}: \Gamma\left(S_{p_{1}}\right) \rightarrow G_{p_{1}}$ is smooth and $G_{p_{1}}$ is nilpotent, by Theorem 8.1 of [7, p. 309] $S_{p_{1}}$ must be nondegenerate. It follows from this that if a Fuchsian group is admissible for supersolubility, then, for the smallest prime $p_{1}$ dividing the order of $G$, the $p_{1}$-local signature $S_{p_{1}}$ of $S$ must be nondegenerate.

Theorem 3.3. Let $\Gamma$ be a finitely generated cocompact Fuchsian group with signature $\left(\gamma ; m_{1}, \ldots, m_{r}\right)$. Suppose $\Gamma(S)$ covers a finite supersoluble group $G$. Then the negative of Euler characteristic of $\Gamma$ satisfies the inequality $Y \geq 1 / 9$, and equality occurs only when $\Gamma$ is the $(2,3,18)$ triangle group with the exception of one Fuchsian group of signature $(0 ; 2,4,6)$ with a negative Euler characteristic of $Y=1 / 12$, which can only cover one supersoluble group of order 24 .

Proof. By the first three cases in the proof of Siegel's theorem [9], if $\gamma \neq 0$ then $Y \geq 1 / 2$ and if $\gamma=0$ and $r \geq 4$, then $Y \geq 1 / 6$. Therefore the only case to be considered here is where $\gamma=0$ and $r=3$, that is among the triangle groups. So $Y=-\chi(S)=1-1 / m_{1}-1 / m_{2}-1 / m_{3}$ where $2 \leq m_{1} \leq m_{2} \leq m_{3}<\infty$. We prove the theorem by considering a few cases, but the fact that $Y>0$ rules out the case of $m_{1}=m_{2}=m_{3}=2$ as well as the case of $m_{1}=m_{2}=2$ and $m_{3} \geq 3$.

Case (1). Suppose all $m_{i} \geq 3$ for $i=1,2,3$. Then

(i) If $m_{1}=m_{2}=m_{3}=3$, then $Y=0$ and $\Gamma$ is not a Fuchsian group.

(ii) If $m_{i} \geq 4$ for all $i=1,2,3$ then $Y \geq 1 / 4$.

(iii) If $m_{1}=3, m_{2} \geq 4, m_{3} \geq 4$, then $Y \geq 1 / 6$.

(iv) If $m_{1}=m_{2}=3, m_{3} \geq 4$, then $Y=1 / 3-1 / m$ and $Y<1 / 9$ only if $m_{3}=4$ and $Y=1 / 12$. But $\Gamma=\Gamma(0 ; 3,3,4)$ by Theorem 3.2 is not admissible for supersolubility because the smallest prime dividing its periods is 2 and its 2-local signature $(0 ; 4)$ is degenerate.

Case (2). $m_{1}=2, m_{2} \geq 3, m_{3} \geq 3$ and $Y=1 / 2-1 / m_{2}-1 / m_{3}$.

(i) If $m_{2} \geq 5, m_{3} \geq 5$ then $Y \geq 1 / 10$. 
(ii) If $m_{2}=4, m_{3} \geq 5$ then $Y<1 / 9$ only when $m_{3} \leq 7$. Hence $m_{3}=5$, 6 and 7 are the only possible numbers for the last period. Therefore we have:

(a) $\Gamma(S)=(0 ; 2,4,5)$ which, by Theorem 3.1 , is not supersoluble-admissible because its derived group $\Gamma^{\prime}$ has the signature $(0 ; 2,5,5)$ which is not nilpotent-admissible or, by Theorem 3.2, simply because, for the smallest prime, 2 , its 2-localization $(0 ; 2,4)$ is degenerate.

(b) $\Gamma(S)=(0 ; 2,4,7)$, by Theorem 3.1 , is not supersoluble-admissible, for its derived group $\Gamma^{\prime}$ has signature $(0 ; 2,7,7)$, which is not nilpotentadmissible, or, by Theorem 3.2 , simply because its 2 -localization $(0 ; 2,4)$ is degenerate.

(c) $\Gamma(S)=(0 ; 2,4,6)$ and $Y=1 / 12$. This is indeed an exceptional case because its derived group $\Gamma^{\prime}$ has the signature $(0 ; 2,2,3,3)$, which is nilpotent-admissible. Therefore Theorem 3.1 does not apply for this subcase. In addition, the smallest prime dividing its periods is 2 , and its 2-local signature $(0 ; 2,2,4)$ is nondegenerate. Therefore Theorem 3.2 does not apply for this subcase either. This means that there is a possibility for a Fuchsian group with the signature $(0 ; 2,4,6)$ to have some supersoluble factors. In the next section we shall see that there is only one candidate for a finite supersoluble group covered by $(0 ; 2,4,6)$ which has order 24 . Actually it is the semidirect product of the dihedral group of order 8 and the cyclic group of order 3 . The structure of this group will be given there.

Case (3). $m_{1}=2$ and $m_{2}=3$ and $Y=\{1 / 6-1 / n, n \geq 7\}$. In this final case $Y \leq 1 / 9$ only when $n \leq 18$, that is $n=7,8,9,10,11,12,13,14,15,16,17$ $\& 18$ and $S=(0,2,3, n)$.

(i) If $(2, n)=(3, n)=1$, then $\Gamma^{\prime}=\Gamma$ and $\Gamma$ is perfect and cannot be residually soluble. This rules out all primes $7,11,13 \& 17$, see [8].

(ii) If $n=2 r$ and $(3, r)=1$, then $\Gamma$ has signature $(2,3,2 r)$, and its derived group $\Gamma^{\prime}$ has signature $(0 ; 3,3, r)$, which is not nilpotent-admissible because, for any prime $p \mid r$ which is naturally different from 3 , the $p$-local signature is degenerate and, by Theorem 3.1, not supersoluble-admissible. This rules out the integers $8,10,14 \& 16$.

(iii) Similarly if $n=3 r$ and $(2, r)=1$ then $\Gamma$ has signature $(0 ; 2,3,3 r)$, where 2 is not a divisor of $r$, and its derived group $\Gamma^{\prime}$ has signature $(0 ; 2,2$, $2, r)$, which is not nilpotent-admissible because if $p \neq 2$ is any prime dividing $r$ then the $p$-local signature of $\Gamma^{\prime}$ is degenerate, thus, by Theorem 3.1, not supersoluble-admissible. This rules out the integers $9 \& 15$. Thus the only candidates for $n$ are $n=12$ and $n=18$, i.e., when $n=6 r, r=2$ or 3. In this final subcase the derived group $\Gamma^{\prime}$ has signature $(1 ; r)$, which has index 6 in $(0 ; 2,3,6 r)$ and is nilpotent-admissible. But, for $r=2$, by Theorem 3.2 , the $(2,3,12)$ group is not admissible for supersolubility because the smallest prime dividing its periods is 2 and its 2 -local signature $(0 ; 2,4)$ is degenerate. Thus, with the exception of one small case when $\Gamma$ has the signature $(0 ; 2,4,6)$, which can cover only one finite supersoluble group of order 24 , 
a bound for a supersoluble-admissible signature occurs when $S$ has the exact form of $(0 ; 2,3,18)$.

Theorem 3.4. Let $\Omega$ be a compact Riemann surface of genus $g \geq 2$, and let $G$ be a finite supersoluble group acting on $\Omega$. Then

(i) If $g=2,|G| \leq 24$, with equality only if $G \simeq D_{4} \otimes \mathbf{Z}_{3}$, with covering the group $(2,4,6)$.

(ii) If $g \geq 3, G$ has order $|G| \leq 18(g-1)$, and equality occurs if and only if $\Omega=H^{2} / \Gamma$, where $\Gamma$ is a proper normal subgroup of finite index in the $(2,3,18)$ triangle group.

Proof. Let $\bar{\Omega}$ be the universal covering space of $\Omega$. Then by a standard argument see $[12$, p. 245], there is a group $\bar{G}$ which covers $G$. This implies there is a smooth homomorphism $\Phi: \bar{G} \rightarrow G$ such that its kernel $\pi_{1}(\Omega)$ is the fundamental group of the surface $\Omega$ and is the group with the signature $(0 ; g)$. Of course, here, $\bar{\Omega}$ is the complex upper half-plane $H^{2}$ and $\bar{G}$ is a Fuchsian group $\Gamma(S)$. Then $\Omega \simeq H^{2} / \pi_{1}(\Omega)$. Moreover, $G$ being the automorphism group of $\Omega$, is isomorphic to $\Gamma(S) / \pi_{1}(\Omega)$. Thus, using the Riemann-Hurwitz identity, we have

$$
|G|=\frac{2(g-1)}{-\chi(S)}
$$

By Theorem 3.3, if $\Gamma$ has signature $(0 ; 2,4,6)$, then $Y=1 / 12$ and $|G|=$ $24(g-1)$. From this it follows that $|G|=24$ only when $g=2$; otherwise $Y \geq 1 / 9$, and equality occurs if and only if $\Gamma$ is the $(2,3,18)$ triangle group. Thus we have

$$
|G|=\frac{2(g-1)}{-\chi(S)} \leq \frac{2(g-1)}{1 / 9}=18(g-1) .
$$

In the next section we will investigate the structure of all finite supersoluble $(2,3,18)$ as well as the unique $(2,4,6)$ triangle groups. We will also answer the question: "Which values of the genus $g$ are possible for a supersoluble automorphism groups of maximal order?"

\section{THE CHIEF SERIES \\ OF THE SUPERSOLUBLE AUTOMORPHISM GROUPS}

In this final section we are due to answer the question: "Which values of $g$ are possible such that a surface of genus $g$ can cover at least one supersoluble automorphism group of maximal order ?" Indeed $g$ is a possible value if and only if $(g-1)=3^{n}$ for all $n=0,2,3, \ldots$ Define $\Gamma_{0}=\Gamma(0 ; 2,4,6)$ and $\Gamma_{1}=\Gamma(0 ; 2,3,18)$, the notations we shall use from now on.

In view of Theorem 3.3 of the last section, which shows that $\Gamma_{0}$ and $\Gamma_{1}$ are the only candidates for a Fuchsian group with a supersoluble-admissible signature and with a Euler characteristic of minimum absolute value, it is natural for us to examine their supersoluble smooth quotient groups. We will also compute the chief series for these groups. 
Theorem 4.1. $\Gamma_{0}=\Gamma(0 ; 2,4,6)$ has a unique supersoluble smooth quotient group $G . \quad G \simeq D_{4} \otimes \mathbf{Z}_{3}$ is the semidirect product of the dihedral group of order 8 and the cyclic group of order 3 admitting a chief series with factors $C_{2}$, $C_{2}, C_{2}$ and $C_{3}$.

Proof. Let $G$ be any finite supersoluble automorphism group covered by $\Gamma_{0}=$ $\left\langle p, q \mid p^{6}=q^{4}=(p q)^{2}=1\right\rangle$. Let $\Phi: \Gamma_{0} \rightarrow G$ be a smooth homomorphism of $\Gamma_{0}$ onto $G$. Then $G$ is generated by two elements $x$ and $y$ satisfying the relators

$$
x^{6}=y^{4}=(x y)^{2}=1 .
$$

Thus 4 and 6 divide the order of $G$. Let $2<3<p_{3}<\cdots<p_{k}$ be the prime factors of the order of $G$. Since $G$ is supersoluble for the $p_{i}$-Sylow subgroups of $G$, we must have

$$
G_{p_{k}} G_{p_{k-1}} \cdots G_{p_{3}} G_{3}=N G_{3} \text { be normal in } G,
$$

where $N=G_{p_{k}} \cdots G_{p_{3}}$ and $G / N G_{3} \simeq G_{2}$. Consider the homomorphism $h: \Gamma_{0} \rightarrow G_{2}$, where $h=n \circ \Phi$ and $n: G \rightarrow G / N G_{3} \simeq G_{2}$ is the natural homomorphism. By Theorem 3.2, $G_{2}$ is a smooth factor group of $\Gamma_{0}$, and the kernel $(h)=N G_{3}$ has an order relatively prime to 2 . The 2-localization of $\Gamma_{0}$ has the signature $S_{2}=(0 ; 2,2,4)$, which is nondegenerate. But it has a positive Euler characteristic of $1 / 4$; therefore, $\Gamma\left(S_{2}\right)$ is finite. Actually it is the dihedral group $D_{4}$ of order 8. Hence, by Theorem (8.1) [7, p. 309], $\Gamma\left(S_{2}\right) \simeq G_{2} \simeq D_{4}$. Abelianizing $\Gamma_{0}$ and letting $\delta: \Gamma_{0} \rightarrow \Gamma_{0} /\left[\Gamma_{0}, \Gamma_{0}\right]$ be the natural map, we can find the signature of $\Gamma_{0}^{\prime}=\left[\Gamma_{0}, \Gamma_{0}\right]$. Since the relators

$$
6 \delta(p)=4 \delta(q)=2 \delta(p+q)=0
$$

yield the relators $2 \delta(p)=2 \delta(q)=0, \Gamma_{0} / \Gamma_{0}^{\prime} \simeq \mathbf{Z}_{2} \oplus \mathbf{Z}_{2}$. Thus, by Singerman's theorem [10], $\Gamma_{0}^{\prime}$ has the signature $(0 ; 2,2,3,3)$, which is nilpotentadmissible. By Theorem 2.7, $G^{\prime}$, the derived group of $G$, is nilpotent and, by the above argument, a factor group of $\Gamma(0 ; 2,2,3,3)$. But the only finite nilpotent factor group of $(0 ; 2,2,3,3)$ is $\mathbf{Z}_{6}$, as one can see easily by localizing this signature. This means that there is only one group which is a candidate for a finite supersoluble group covered by $(0 ; 2,4,6)$, and it must have order 24 . This final analogy also proves that $N G_{3}$, the kernel of $h$, is a cyclic subgroup of order 3, i.e., $N G_{3} \simeq \mathbf{Z}_{3}$ which is also normal in $G$. It is now clear that $G$ must be the semidirect product of $D_{4}$ and $Z_{3}$, and it is unique. To check its supersolubility we actually construct this $(2,4,6)$ automorphism group in the following subsection.

Construction of the unique $(2,4,6)$ supersoluble automorphism group and its chief series. Let $D_{4}=\left\langle a, b \mid a^{2}=b^{4}=(a b)^{2}=1\right\rangle$ be the dihedral group of order 8. Let $\mathbf{Z}_{3}=\left\langle z \mid z^{3}=1\right\rangle$ be the cyclic group of order 3 and the action be given by the relations $b z b^{-1}=z^{-1}, a z=z a$. Then, letting $x=a z$ and $y=b$ be the generators for $G=\Gamma(0 ; 2,4,6)$, we have the following relations:

$$
x^{2}=(a z)^{2}=z^{2}=z^{-1} \text {, }
$$


and so $z=x^{-2}$, which implies $a=x z^{-1}=x^{3}$ and $b=y$.

Now $x y=a z b$, that is, $(x y)^{2}=a z b a z b=a b z^{-1} z a b=(a b)^{2}=1$.

Thus $G=\left\langle x, y \mid x^{6}=y^{4}=(x y)^{2}=1\right\rangle$, this is, $G$ is a $(2,4,6)$-group. Moreover $\mathbf{Z}_{3}$ is normal in $G$ but $D_{4}$ is not normal. Therefore $G \simeq D_{4} \otimes \mathbf{Z}_{3}$.

Finally a chief series for this unique exceptional supersoluble group is given by the following chains of subgroups:

$$
\Gamma(0 ; 2,4,6) \triangleright \Gamma(0 ; 3,4,4) \triangleright \Gamma(0 ; 2,2,3,3) \triangleright \Gamma(0 ; 3,3,3,3) \triangleright \Gamma(2 ;)
$$

or, equivalently,

$$
D_{4} \otimes \mathbf{Z}_{3} \triangleright \mathbf{Z}_{3} \otimes \mathbf{Z}_{4} \triangleright \mathbf{Z}_{6} \triangleright \mathbf{Z}_{3} \triangleright\{1\},
$$

where $\mathbf{Z}_{3} \otimes \mathbf{Z}_{4}$ is the semidirect product of the cyclic groups $\mathbf{Z}_{3}$ and $\mathbf{Z}_{4}$ and has the presentation

$$
\mathbf{Z}_{3} \otimes \mathbf{Z}_{4}=\left\langle a^{6}=1, a^{3}=b^{2}=(a b)^{2}\right\rangle=\left\langle x=a b, y=b \mid x^{4}=y^{4}=(x y)^{3}=1\right\rangle .
$$

The series has the chief factors $C_{2}, C_{2}, C_{2}$, and $C_{3}$, all cyclic and of prime orders. Therefore, if a surface of genus $g$ admits a supersoluble automorphism group $G$ of order $24(g-1)$, then $g$ must be equal to 2 and there exists only one supersoluble automorphism group covered by $\Gamma_{0}=\Gamma_{0}(0 ; 2,4,6)$, which is this given semidirect product. In the next theorem we will investigate the structure of all other supersoluble automorphisms of maximal order, but first we need to give the following technical lemma.

Lemma 4.2. For any two distinct primes $p$ and $q$, a group $G$ of order $p q^{n}$, for which $p \mid(q-1)$ is always supersoluble.

Proof. Using the Sylow theory, a Sylow $q$-subgroup $G_{q}$ of $G$ must be normal in $G$. Also, since $G / G_{q} \simeq \mathbf{Z}_{p}$ is the unique cyclic group of order $p$ that is abelian and of exponent dividing $q-1, G$ is strictly $p$-closed. Thus, by Theorem 2.9, $G$ is supersoluble.

Theorem 4.3. Let $\Gamma_{1}=\Gamma(0 ; 2,3,18)$. Let $\Omega$ be any surface of genus $g$ admitting a supersoluble automorphism group $G$ of order $18(g-1)$. Then $G$ can be covered by $\Gamma_{1}$ if and only if $(g-1)=3^{n}$ for all $n \geq 2$.

Proof. The argument in Theorem 3.4 shows that if $|\operatorname{Aut}(\Omega)|=18(g-1)$, then $\Omega \simeq H^{2} / \Gamma$, where $\Gamma$ is a proper normal subgroup of finite index in $\Gamma_{1}$. Here $\Gamma$ is defined to be the group of covering transformations of the universal covering map $\theta: H^{2} \rightarrow \Omega$. We prove the theorem in two steps.

(a) Suppose $G$ of order $18(g-1)$ is covered by $\Gamma_{1}$. Then there exists a smooth homomorphism, $\Phi: \Gamma(0 ; 2,3,18) \rightarrow G$, onto $G$. Thus $G$ must be generated by two elements $P$ and $Q$ satisfying the relations

$$
P^{2}=Q^{3}=(P Q)^{18}=1 \text {. }
$$

If $G$ is supersoluble then its order $|G|$ must be divisible by the first two primes, namely 2 and 3 . As usual let $2<3<p_{3} \cdots<p_{k}$ be in this ordering 
all the prime factors of $|G|$. Suppose $G_{2}, G_{3}, G_{p_{3}}, \ldots, G_{p_{k}}$ are the corresponding Sylow subgroups. Then, by the Sylow tower property of $G$, we have $G \triangleright G_{p_{k}} G_{p_{k-1}} \cdots G_{3}=N G_{3}$, where as before $N=G_{p_{k}} \cdots G_{p_{3}}$ and $G / N G_{3} \simeq G_{2}$. The 2-localization of the signature $S=(0 ; 2,3,18)$ is actually $S_{2}=(0 ; 2,2)$, which is nondegenerate but has the positive Euler characteristic $\chi\left(S_{2}\right)=1$. Therefore, by Corollary 6.7 in [7, p. 307], $G_{2} \simeq \Gamma\left(S_{2}\right) \simeq \mathbf{Z}_{2}$. Let, as before, $n: G \rightarrow G / N G_{3} \simeq G_{2}$ be the natural homomorphism. Consider the homomorphism $f: \Gamma_{1} \rightarrow G_{2}$ : then $f=n \circ \Phi$ and $\operatorname{ker}(f) \simeq N G_{3}$ has an order relatively prime to 2 . Next, by Singerman's theorem [10], $\operatorname{ker}(f) \simeq \Gamma(0 ; 3,3,9)$, moreover $\left|N G_{3}\right|=9(g-1)$. Thus, by Theorem 1.1.2 [13, p. 239], $N G_{3}$, being a factor group of $\Gamma(0 ; 3,3,9)$, must be a nilpotent 3-group. Thus, by $\S 1.3$ of [13, p. 240], if a surface of genus $g$ admits a nilpotent automorphism group of order $9(g-1)$ (the order of maximal automorphism 3-groups), then $(g-1)$ must be a power of 3 . This means $(g-1)=3^{n}$, and so $|G|=2 \cdot 3^{n+2}$ for all $n \geq 2$.

(b) Conversely, if $(g-1)=3^{n}$, then the order of $G$ will be given by $|G|=$ $18\left(3^{n}\right)=2 \cdot 3^{n+2}$, and since $2 \mid(3-1)$ by Lemma $4.2, G$ must be supersoluble.

In a separate paper entitled "Classification of $p$-groups of automorphisms of Riemann surfaces and their lower central series" by this author [13] it is proved that the maximal 3-groups of automorphisms of Riemann surfaces of genus $g \geq 2$ have order $9(g-1)$ and that this upper bound occurs when the Fuchsian group $\Gamma$ has the exact signature of $(0 ; 3,3,9)$.

Thus Theorem 4.3 also proves that the maximal automorphism 3-groups are normal subgroups of index 2 in the maximal supersoluble automorphism groups. We also have the following theorem on the existence of a supersoluble automorphism group.

Theorem 4.4. For any integer $n \geq 4$, there exists a supersoluble automorphism group $G$ of order $2 \cdot 3^{n}$ acting on a compact Riemann surface $\Omega$ of genus $g=$ $3^{n-2}+1$.

Proof. By Theorem 1.3.1 in [13, p. 241] for any integer $n \geq 4$, there exists a nilpotent automorphism 3-group $G_{3}$ of order $3^{n}$ that can act on a compact Riemann surface $\Omega$ of genus $g=3^{n-2}+1$. By the argument in the same paper, $G_{3}$ is a $(3,3,9)$ group, that is a normal subgroup of index 2 in the supersoluble $(2,3,18)$ groups. Therefore $G_{3}$ must admit an automorphism of order 2 which permutes the two conjugacy classes of the periodic generators of order 3 cyclically.

Thus there exists a $(2,3,18)$ group of order $18(g-1)=2 \cdot 3^{n}$ which can be obtained by attaching that element of order 2 which permutes these two periodic generators in this manner.

Construction of a $(2,3,18)$ supersoluble automorphism group and its chief series. Let $\Gamma_{1}=\Gamma(0 ; 2,3,18)=\left\langle p, q \mid p^{2}=q^{3}=(p q)^{18}=1\right\rangle$. Let $\tau: \Gamma_{1} \rightarrow$ 
$\Gamma_{1} /\left[\Gamma_{1}, \Gamma_{1}\right]$ be the abelianizing homomorphism, where $\left[\Gamma_{1}, \Gamma_{1}\right]=\Gamma_{1}^{\prime}$ is the derived group of $\Gamma_{1}$. Thus we have $2 \tau(p)=3 \tau(q)=18(\tau(p)+\tau(q))=0$.

But it is easy to see that these relators yield

$$
\Gamma_{1} / \Gamma_{1}^{\prime} \simeq \mathbf{Z}_{2} \otimes \mathbf{Z}_{3} \simeq \mathbf{Z}_{6} .
$$

By Singerman's theorem [10], $\Gamma_{1}^{\prime}$ has signature $(1 ; 3)$ which is nilpotentadmissible. Now since $\Gamma_{1}^{\prime}$ has index 6 in $\Gamma_{1}$, it is more convenient to take the intermediate step via the subgroup $\operatorname{ker}(\theta)$ in $\Gamma_{1}$ of index 2 , where $\theta: \Gamma_{1} \rightarrow \mathbf{Z}_{2}$ is the homomorphism defined by $\theta(p) \equiv 1(\bmod 2), \theta(q) \equiv 0(\bmod 2)$. Then $\Gamma_{1} \supset \operatorname{ker}(\theta) \supset \Gamma_{1}^{\prime}$ and $\operatorname{ker}(\theta) / \Gamma_{1}^{\prime} \simeq\langle\tau(q)\rangle$ has order 3 .

A Schreier set of coset representatives for $\operatorname{ker}(\theta)$ in $\Gamma_{1}$ is $U=\{1, p\}$. Clearly $q \in \operatorname{ker}(\theta)$, but $p \notin \operatorname{ker}(\theta)$. Therefore the generators and relations of $\operatorname{ker}(\theta)$ are obtained by the following tables, where $X$ and $R$ represent the set of generators and relators respectively.

[GENERATORS]

\begin{tabular}{|l|l|l|}
\hline$U \backslash X$ & $p$ & $q$ \\
\hline 1 & 1 & $q$ \\
$p$ & $p^{2}$ & $p q p$ \\
\hline
\end{tabular}

(4.1)
[RELATIONS]

\begin{tabular}{|l|l|l|l|}
\hline$U \backslash R$ & $p^{2}$ & $q^{3}$ & $(p q)^{18}$ \\
\hline 1 & $p^{2}$ & $q^{3}$ & $(p q)^{18}$ \\
$p$ & $p^{2}$ & $p q^{3} p$ & $p(p q)^{18} p$ \\
\hline
\end{tabular}

Letting $\alpha=p q p, \beta=q$, and $\varepsilon=p^{2}$ and rewriting the relators in terms of these new generators, we have:

$$
\begin{aligned}
p^{2}=1 & \sim \varepsilon=1 \\
q^{3}=1 & \sim \beta^{3}=1 \\
(p q)^{18}=1 & \sim(\alpha \beta)^{9}=1 \\
p q^{3} p=1 & \sim \alpha^{3}=1 \\
p(p q)^{18} p=1 & \sim(\beta \alpha)^{9}=1 .
\end{aligned}
$$

Thus

$$
\Gamma_{2}=\left\langle\alpha, \beta \mid \alpha^{3}=\beta^{3}=(\alpha \beta)^{9}=1\right\rangle=\operatorname{ker}(\theta),
$$

which is ultimately a $(3,3,9)$ group of order $9(g-1)$. Referring to the above mentioned paper [13], it is proved that the genus $g$ has the minimum value of 10. Burnside [1] shows there is a $(3,3,9)$ group of order $3^{4}$ that is a nilpotent 3-group with the presentation

$$
H_{1}=\left\langle x, y \mid x^{3}=y^{3}=(x y)^{9}=\left(x y^{-1}\right)^{3}=1,(x y)^{3}=(y x)^{3}\right\rangle .
$$

Let $\mathbf{Z}_{2}=\left\langle z \mid z^{2}=1\right\rangle$, and let the action on the group be given by

$$
z y z=x, \text { and } z x z=y \text {. }
$$


Let the new generators of the super group be given by

$$
p=z \text { and } q=x .
$$

Then we have the relations

$$
p^{2}=q^{3}=1, \text { and }(p q)^{2}=z x z x=y x,
$$

thus $(p q)^{18}=(y x)^{9}=(x y)^{9}=1$.

But the extra relations must also be written in terms of these new generators, so we have $x=q, y=z x z=p q p$ and obtain the extra relations

$$
\left(x y^{-1}\right)^{3}=1 \sim\left(q p q^{-1} p\right)^{3}=1 \text { or }\left(p q p q^{-1}\right)^{3}=1
$$

and the relation

$$
(x y)^{3}=(y x)^{3} \sim(p q)^{6}=(q p)^{6} .
$$

Therefore a supersoluble smooth factor group for the genus $g=10$ has the presentation

$$
G_{1}=\left\langle p, q \mid p^{2}=q^{3}=(p q)^{18}=[p, q]^{3}=1,(p q)^{6}=(q p)^{6}\right\rangle,
$$

which is clearly supersoluble by Lemma 4.2 since it has an order of $162=2 \cdot 3^{4}$.

A chief series for this maximal automorphism supersoluble group which occurs when $g=10$ is given by the following chain of normal subgroups:

$$
G_{1} \triangleright H_{1} \triangleright H_{2} \triangleright H_{3} \triangleright H_{4} \triangleright\{1\},
$$

where $G_{1}$ and $H_{1}$ are as above and $H_{2}, H_{3}, H_{4}$ are the other normal subgroups in this chief series, all normal in $G_{1}$. Equivalently, if we present these subgroups according to their signature representations, we will obtain

$$
\begin{aligned}
\Gamma(0 ; 2,3,18) \triangleright \Gamma(0 ; 3,3,9) \triangleright \Gamma(0 ; 9,9,9) \\
\\
\qquad \Gamma(1 ; 3,3,3) \triangleright \Gamma(1 ; 3,3,3,3,3,3,3,3,3) \triangleright \Gamma(10 ;),
\end{aligned}
$$

which has the cyclic factor groups of prime orders $C_{2}, C_{3}, C_{3}, C_{3}$, and $C_{3}$.

\section{ACKNOWLEDGMENTS}

I am indebted to Professor R. S. Kulkarni for drawing my attention to some of his recent work [4] which appears to have some overlaps with the results obtained in [13] as well as some overlap among [7] and [5], see [4], [5] in particular. The author also wishes to thank Professor A. M. Macbeath for all his help in finding the exceptional case of the results, and Miriam E. Lindauer for all her help with the computer for this paper.

\section{REFERENCES}

1. W. Burnside, Theorem of groups of finite order, Note $K$, Dover, New York, 1955.

2. H. S. M. Coxeter, W. O. J. generators and relations for discrete groups, Ergebnisse der Math., Bd. 14, 3rd. ed., Springer, Berlin-Heidelberg-New York, 1972.

3. K. W. Gruenberg, Residual properties of infinite soluble groups, Proc. London Math. Soc. (3) 7 (1957), 29-62. 
4. R. S. Kulkarni, Symmetries of surfaces, Topology 26 (1987), 195-203.

5. __ Normal subgroups of Fuchsian groups, Quart. J. of Math. 36 (1985), 325-344.

6. A. M. Macbeath, On a theorem of Hurwitz, Glasgow Math. J. 5 (1961), 90-96.

7. __ Residual nilpotency of Fuchsian groups, Illinois J. Math. (2) 28 (1984), 299-311.

8. C. H. Sah, Groups related to compact Riemann surfaces, Acta Math. 123 (1969), 13-42.

9. C. L. Siegel, Some remarks on discontinuous groups, Annals of Math. 46 (1945), 708-718.

10. D. Singerman, Subgroups of Fuchsian groups and finite permutation groups, Bull. London Math. Soc. 2 (1970), 319-323.

11. M. Weinstein, ed., Between nilpotent and soluble, House, Passaic, New Jersey, 1982, 2-42.

12. R. Zomorrodian, Nilpotent automorphism groups of Riemann surfaces, Trans. Amer. Math. Soc. 288 (1985), 241-255.

13. _ Classification of p-groups of automorphisms of Riemann surfaces and their lower central series, Glasgow Math. J. 29 (1987), 237-244.

Department of Mathematics and Statistics, University of Pittsburgh, Pittsburgh, Pennsylvania 15260

Current address: Department of Mathematics, Pennsylvania State University, McKeesport, Pennsylvania 15132 